\title{
Mahler's classification of numbers compared with Koksma's
}

\author{
by \\ YAnn Bugeaud (Strasbourg)
}

1. Introduction. Mahler [7], in 1932, and Koksma [6], in 1939, introduced two related measures of the degree of approximation of a complex transcendental number $\xi$ by algebraic numbers. For any integer $n \geq 1$, we denote by $w_{n}(\xi)$ the supremum of the exponents $w$ for which

$$
0<|P(\xi)|<\mathrm{H}(P)^{-w}
$$

has infinitely many solutions in integer polynomials $P(X)$ of degree at most $n$. Here, $\mathrm{H}(P)$ stands for the naïve height of the polynomial $P(X)$, that is, the maximum of the absolute values of its coefficients. Further, we set $w(\xi)=\limsup _{n \rightarrow \infty}\left(w_{n}(\xi) / n\right)$ and, according to Mahler [7], we say that $\xi$ is an

- $S$-number if $w(\xi)<\infty$;

- T-number if $w(\xi)=\infty$ and $w_{n}(\xi)<\infty$ for any integer $n \geq 1$;

- $U$-number if $w(\xi)=\infty$ and $w_{n}(\xi)=\infty$ for some integer $n \geq 1$.

In the sense of the Lebesgue measure, almost all numbers are $S$-numbers. Liouville numbers are examples of $U$-numbers, but the existence of $T$-numbers remained an open problem during nearly forty years, until it was confirmed by Schmidt [10, 11].

Following Koksma [6], for any integer $n \geq 1$, we denote by $w_{n}^{*}(\xi)$ the supremum of the exponents $w$ for which

$$
0<|\xi-\alpha|<\mathrm{H}(\alpha)^{-w-1}
$$

has infinitely many solutions in complex algebraic numbers $\alpha$ of degree at most $n$. Here, $\mathrm{H}(\alpha)$ stands for the naïve height of $\alpha$, that is, the naïve height of its minimal defining polynomial. Koksma [6] defined $S^{*}$, $T^{*}$ - and $U^{*}$ numbers as above, using $w_{n}^{*}$ in place of $w_{n}$. He proved that this classification of numbers is equivalent to Mahler's (see e.g. the book of Schneider [14]).

2000 Mathematics Subject Classification: Primary 11J04. 
For more information on the functions $w_{n}$ and $w_{n}^{*}$, the reader is directed to Wirsing [16] and Schmidt [13].

For any integer $n \geq 2$ and any complex transcendental number $\xi$ we have

$$
w_{n}^{*}(\xi) \leq w_{n}(\xi) \leq w_{n}^{*}(\xi)+n-1 .
$$

The first inequality in (1) is easy (see e.g. [12, p. 44]), and the second one is due to Wirsing [16]. Thus, it is a natural question to ask whether there are complex numbers $\xi$ such that $w_{n}^{*}(\xi)<w_{n}(\xi)$ for some integer $n \geq 2$. In 1976, R. C. Baker [1] gave a positive answer to this problem by proving that for any integer $n \geq 2$ the function $w_{n}-w_{n}^{*}$ can take any value in the interval $[0,(n-1) / n]$. He even succeeded in constructing real numbers $\xi$ with prescribed values for $w_{n}(\xi)$ and $w_{n}^{*}(\xi)$ for all positive integers $n$.

In the present work, we improve upon Baker's result: we show that for any integer $n \geq 3$ the set of values taken by the function $w_{n}-w_{n}^{*}$ contains the interval $[0, n / 4]$. As in [1], our method of proof originates in two papers by Schmidt [10,11], where the existence of $T$-numbers is established. The main novelty introduced here is the use of integer polynomials having two zeros very close to each other.

Our results are stated in Section 2 and proved in Sections 5, 6 and 7. Further related comments are made in Section 8. Section 4 is devoted to auxiliary lemmas, and an independent remark on Koksma's classification is the purpose of Section 3.

Acknowledgements. I would like to thank the referee for his very careful reading of the manuscript.

2. The main result. Theorem 1 asserts the existence of real numbers with special properties.

Theorem 1. Let $n \geq 3$ be an integer and set $F(n)=2 n^{3}+2 n^{2}+3 n-1$. Let $w_{n}$ and $w_{n}^{*}$ be real numbers such that

$$
w_{n}^{*} \leq w_{n} \leq w_{n}^{*}+n / 4, \quad w_{n}>F(n) .
$$

Then there exists a real number $\xi$ such that

$$
w_{n}^{*}(\xi)=w_{n}^{*} \quad \text { and } \quad w_{n}(\xi)=w_{n} .
$$

As in [1], $\xi$ is obtained as the limit of a suitable sequence $\xi_{j}=\left(c_{j}+\gamma_{j}\right) / g_{j}$ of algebraic numbers, where the $c_{j}$ 's and $g_{j}$ 's are positive integers and the $\gamma_{j}$ 's are real algebraic numbers of degree $n$. Thanks to a rather tedious and complicated construction, the differences $\left|\xi-\xi_{j}\right|$ are precisely controlled and $w_{n}^{*}(\xi)$ satisfies

$$
w_{n}^{*}(\xi)=\lim _{j \rightarrow \infty} \frac{-\log \left|\xi-\xi_{j}\right|}{\log \mathrm{H}\left(\xi_{j}\right)}-1 .
$$


Our new idea is to take for the $\xi_{j}$ 's algebraic numbers having a complex conjugate $\xi_{j}^{\sigma}$ very close to them. It then follows that $\left|\xi-\xi_{j}^{\sigma}\right|$ is very small and that $\left|P_{j}(\xi)\right|$ is much smaller than $\left|\mathrm{H}\left(\xi_{j}\right)\right|^{-w_{n}^{*}(\xi)}$, where $P_{j}(X)$ denotes the minimal defining polynomial of $\xi_{j}$. Consequently, $w_{n}(\xi)$ is larger than $w_{n}^{*}(\xi)$. Since a few important changes are needed in the argument of [1], we give the full details of the proof of Theorem 1 . We point out that our method is effective, which is not the case of that of [1]; see Section 8 for explanations.

The fact that the function $n \mapsto F(n)$ occurring in the statement of Theorem 1 is of order of magnitude $n^{3}$ is due to technical constraints. Presumably, the same result holds true also when $F$ is much smaller. Notice that Baker [1] proved Theorem 1 with (2) replaced by

$$
w_{n}^{*} \leq w_{n} \leq w_{n}^{*}+(n-1) / n, \quad w_{n}>n^{3}+2 n^{2}+5 n+1,
$$

and that Theorem 1 also holds when (2) is replaced by

$$
w_{n}^{*}+(n-1) / n \leq w_{n} \leq w_{n}^{*}+n / 4, \quad w_{n}>2 n^{3}+n-1,
$$

as will be clear from the proof.

However, the upper bound in (1) can be lowered when $w_{n}(\xi)$ is close to $n$. Namely, Wirsing [16] proved that for any integer $n \geq 2$ and any real transcendental number $\xi$ we have

$$
w_{n}(\xi) \leq w_{n}^{*}(\xi)\left(w_{n}(\xi)-n+1\right),
$$

which is sharper than (1) for

$$
w_{n}(\xi) \leq \frac{n+\sqrt{n^{2}+4 n-4}}{2} .
$$

It turns out that our method allows us to construct real numbers $\xi$ with prescribed values for $w_{n}(\xi)$ and $w_{n}^{*}(\xi)$, for finitely many integers $n$. Suitable modifications of the proof of Theorem 1 yield the following result.

Theorem 2. Let $3 \leq n_{1}<\ldots<n_{k}$ be positive integers. Let $w_{1}^{*} \leq \ldots \leq$ $w_{k}^{*}$ and $w_{1} \leq \ldots \leq w_{k}$ be real numbers satisfying

$$
w_{j}^{*} \leq w_{j} \leq w_{j}^{*}+n_{j} / 4, \quad w_{j}>2 n_{j}^{3}+2 n_{j}^{2}+3 n_{j}-1 \quad(1 \leq j \leq k) .
$$

Then the set of real $S$-numbers $\xi$ such that

$$
w_{n_{j}}^{*}(\xi)=w_{j}^{*} \quad \text { and } \quad w_{n_{j}}(\xi)=w_{j} \quad \text { for any } 1 \leq j \leq k,
$$

has positive Hausdorff dimension.

Since the proof of Theorem 1 is already very technical, we do not give a complete proof of Theorem 2 . We merely describe and explain which changes are to be done. This is the content of Section 7. For an introduction to the theory of Hausdorff dimension, the reader is directed e.g. to the book of Falconer [3]. 
Finally, we would like to propose an open problem. For an $S$-number $\xi$, we define its type $t(\xi)$ and its $*$-type $t^{*}(\xi)$ by $t(\xi)=\limsup _{n \rightarrow \infty} w_{n}(\xi) / n$ and $t^{*}(\xi)=\limsup _{n \rightarrow \infty} w_{n}^{*}(\xi) / n$, respectively. We infer from (1) that $t^{*}(\xi) \leq$ $t(\xi) \leq t^{*}(\xi)+1$.

Problem. Do there exist real numbers $\xi$ with $t^{*}(\xi)<t(\xi)$, that is, with

$$
\limsup _{n \rightarrow \infty} \frac{w_{n}^{*}(\xi)}{n}<\limsup _{n \rightarrow \infty} \frac{w_{n}(\xi)}{n} ?
$$

3. A remark on Koksma's classification. The number $w_{n}^{*}(\xi)$ is defined by taking into account all the algebraic numbers which are close to $\xi$. However, when $\xi$ is a real transcendental number, it would be more natural to consider only the real algebraic numbers which are close to $\xi$. The aim of Lemma 1 below is to prove that this makes however no difference. For integers $n \geq 1$ and $H \geq 1$, set

$$
\begin{aligned}
w_{n}^{* r}(H, \xi) & :=\min \{|\xi-\alpha|: \alpha \text { real algebraic, } \operatorname{deg}(\alpha) \leq n, \mathrm{H}(\alpha) \leq H, \alpha \neq \xi\}, \\
w_{n}(H, \xi) & :=\min \{|\xi-\alpha|: \alpha \text { algebraic, } \operatorname{deg}(\alpha) \leq n, \mathrm{H}(\alpha) \leq H, \alpha \neq \xi\}, \\
w_{n}^{* r}(\xi) & :=\limsup _{H \rightarrow \infty} \frac{-\log \left(H w_{n}^{* r}(H, \xi)\right)}{\log H} .
\end{aligned}
$$

It is easy to check that

$$
w_{n}^{*}(\xi)=\limsup _{H \rightarrow \infty} \frac{-\log \left(H w_{n}^{*}(H, \xi)\right)}{\log H} .
$$

Further, we have the inequality

$$
w_{n}^{* r}(\xi) \leq w_{n}^{*}(\xi),
$$

which turns out to be an equality, as stated in the next lemma.

LEMMA 1. For any integer $n \geq 1$ and any real transcendental number $\xi$, we have $w_{n}^{* r}(\xi)=w_{n}^{*}(\xi)$. Consequently, in order to determine $w_{n}^{*}(\xi)$, it is enough to consider the real algebraic numbers which are close to $\xi$.

Proof. The idea of the proof is due to Maurice Mignotte. Let $n \geq 1$ be an integer, $H>1$ be a real number and $\xi$ be a real transcendental number. Let $\alpha_{1}$ be an algebraic number of height at most $H$ and of degree $n_{1} \leq n$ such that $w_{n}^{*}(H, \xi)=\left|\xi-\alpha_{1}\right|$. We may assume that $\alpha_{1}$ is non-real, otherwise the lemma is clearly true. Then the minimal defining polynomial of $\alpha_{1}$, denoted by $P_{1}(X)$, has two distinct roots $\alpha_{1}$ and $\bar{\alpha}_{1}$ very near to $\xi$. Grace's complex version of Rolle's theorem (see e.g. [2, p. 25]) asserts that its derivative $P_{1}^{\prime}(X)$ has a root $\alpha_{2}$ in the closed disk centered at $\left(\alpha_{1}+\bar{\alpha}_{1}\right) / 2$ and of radius $\left|\alpha_{1}-\bar{\alpha}_{1}\right| \cot \left(\pi / n_{1}\right) / 2$. Observe that this closed disk reduces 
to the point $\left(\alpha_{1}+\bar{\alpha}_{1}\right) / 2$ if $n_{1}=2$. Consequently, we have

$$
\begin{gathered}
\left|\xi-\alpha_{2}\right| \leq\left|\xi-\operatorname{Re} \alpha_{1}\right|+\frac{\left|\alpha_{1}-\bar{\alpha}_{1}\right|}{2} \cdot \frac{n}{2} \leq\left(\frac{n}{2}+1\right)\left|\xi-\alpha_{1}\right|, \\
\operatorname{deg}\left(\alpha_{2}\right) \leq n_{1}-1 \text { and } \mathrm{H}\left(\alpha_{2}\right) \leq 2^{n} \mathrm{H}\left(P_{1}^{\prime}\right) \leq 2^{n} n H .
\end{gathered}
$$

Indeed, the minimal defining polynomial of $\alpha_{2}$ is a divisor of $P_{1}^{\prime}(X)$, hence its height is less than or equal to $2^{\operatorname{deg}\left(P_{1}^{\prime}\right)} \mathrm{H}\left(P_{1}^{\prime}\right)$, by using the "Gelfond inequality", as stated e.g. in [15, Remark 2, p. 81]. If $\alpha_{2}$ is non-real, we proceed further in the same way in order to construct an algebraic approximant $\alpha_{3}$ of $\xi$ whose degree is strictly less than the degree of $\alpha_{2}$. We iterate this process as soon as we end up with a real approximant. This always happens since the degrees of the algebraic numbers we construct are strictly decreasing. Consequently, there exists a real number $\alpha$ with

$$
\mathrm{H}(\alpha) \leq\left(2^{n} n\right)\left(2^{n-1}(n-1)\right) \ldots(2 H) \leq 2^{n^{2}} n^{n} H
$$

and

$$
|\xi-\alpha| \leq\left(\frac{n}{2}+1\right) \ldots\left(\frac{2}{2}+1\right)\left|\xi-\alpha_{1}\right| \leq n^{n}\left|\xi-\alpha_{1}\right| .
$$

Thus, for any real number $H \geq 1$ we have

$$
w_{n}^{* r}\left(2^{n^{2}} n^{n} H, \xi\right) \leq n^{n} w_{n}^{*}(H, \xi)
$$

and

$$
w_{n}^{*}(\xi) \leq \limsup _{H \rightarrow \infty} \frac{-\log \left(H n^{-n} w_{n}^{* r}\left(2^{n^{2}} n^{n} H, \xi\right)\right)}{\log H} \leq w_{n}^{* r}(\xi),
$$

as asserted.

REMARK. The idea of the proof of Lemma 1 can also be applied to approximation in the $p$-adic field $\mathbb{Q}_{p}$. Recall that this field is not algebraically closed, and denote by $\overline{\mathbb{Q}}_{p}$ an algebraic closure of it. We can show that for any integer $n \geq 1$ and any transcendental number $\xi$ in $\mathbb{Q}_{p}$ the supremum of the exponents $w$ for which

$$
0<|\xi-\alpha|<\mathrm{H}(\alpha)^{-w-1}
$$

has infinitely many solutions in algebraic numbers $\alpha$ in $\overline{\mathbb{Q}}_{p}$ of degree at most $n$ is equal to the supremum of the exponents $w$ for which the same inequality has infinitely many solutions in algebraic numbers $\alpha$ in $\mathbb{Q}_{p}$. Indeed, let $H>1$ be a real number and let $\alpha_{1}$ be an algebraic number in $\overline{\mathbb{Q}}_{p}$ of height at most $H$ and degree $n_{1} \leq n$, such that

$\left|\xi-\alpha_{1}\right|=\min \left\{|\xi-\alpha|: \alpha\right.$ algebraic in $\left.\overline{\mathbb{Q}}_{p}, \operatorname{deg}(\alpha) \leq n, \mathrm{H}(\alpha) \leq H, \alpha \neq \xi\right\}$.

We may assume that $\alpha_{1}$ is not in $\mathbb{Q}_{p}$, otherwise there is nothing to do. Denote by $\alpha_{1}^{(1)}:=\alpha_{1}, \alpha_{1}^{(2)}, \ldots, \alpha_{1}^{\left(n_{1}\right)}$ the conjugates of $\alpha_{1}$, numbered in such a way 
that

$$
\left|\xi-\alpha_{1}\right| \leq\left|\xi-\alpha_{1}^{(2)}\right| \leq \ldots \leq\left|\xi-\alpha_{1}^{\left(n_{1}\right)}\right| .
$$

If $\left|\xi-\alpha_{1}\right|<\left|\xi-\alpha_{1}^{(2)}\right|$, then Krasner's Lemma (see e.g. [9, p. 130]) implies that $\alpha_{1}$ lies in $\mathbb{Q}_{p}$, which we have excluded. Consequently, the minimal defining polynomial $P_{1}(X)$ of $\alpha_{1}$ has two roots $\alpha_{1}$ and $\alpha_{1}^{(2)}$ with $\left|\xi-\alpha_{1}\right|=\left|\xi-\alpha_{1}^{(2)}\right|$, and we deduce from the $p$-adic version of Rolle's theorem (see e.g. [9, p. 316]) that $P_{1}^{\prime}(X)$ has a root $\alpha_{2}$ with $\mathrm{H}\left(\alpha_{2}\right) \leq 2^{n} \mathrm{H}\left(P_{1}^{\prime}\right) \leq 2^{n} n H$ and $\left|\xi-\alpha_{2}\right|<$ $p^{2}\left|\xi-\alpha_{1}\right|$. If $\alpha_{2}$ does not lie in $\mathbb{Q}_{p}$, we iterate this process, exactly as in the proof of Lemma 1.

4. Auxiliary results. Lemma 2 below gives a version of the Liouville inequality.

Lemma 2. Let $\alpha$ and $\beta$ be distinct algebraic numbers of degree at most $m$ and $n$, respectively. Then there exists a positive constant $c(m, n)<1$, depending only on $m$ and $n$, such that

$$
|\alpha-\beta| \geq c(m, n) \mathrm{H}(\alpha)^{-n} \mathrm{H}(\beta)^{-m} .
$$

An admissible value for $c(m, n)$ is $(m+1)^{-n-1}(n+1)^{-m-1}$.

Proof. This is a direct consequence of Theorems 6 and 7 of Güting [4].

In the next lemma we define a two-parameter infinite family of integer polynomials (found by Mignotte [8]) having two zeros very close to each other.

Lemma 3. Let $n \geq 3$ and $a \geq 10$ be integers. The polynomial

$$
P_{n, a}(X):=X^{n}-2(a X-1)^{2}
$$

is irreducible and has two real roots very close to each other, namely

$$
\begin{aligned}
& \delta^{+}(n, a):=a^{-1}+a^{-(n+2) / 2} / \sqrt{2}+\varepsilon^{+}(n, a), \\
& \delta^{-}(n, a):=a^{-1}-a^{-(n+2) / 2} / \sqrt{2}+\varepsilon^{-}(n, a),
\end{aligned}
$$

where $\left|\varepsilon^{+}(n, a)\right|,\left|\varepsilon^{-}(n, a)\right| \leq C(a / 2)^{-n-1}$ for some absolute constant $C$. Further, it follows from Rouché's theorem that $P_{n, a}(X)$ has no other roots in the disk centered at the origin and of radius $1 / 2$.

Proof. The irreducibility of $P_{n, a}(X)$ follows from the Eisenstein criterion, and Rouché's theorem shows that $P_{n, a}(X)$ has exactly two roots in the disk centered at the origin and of radius $1 / 2$. Studying the function $x \mapsto P_{n, a}\left(a^{-1}+x\right)$ in a neighbourhood of the origin, we see that these two roots can be expressed as stated above. 
LEMMA 4. Let $\alpha$ be an algebraic number of degree $n \geq 1$ and let $a, b$ and $c$ be integers with $c \neq 0$. Then

$$
\mathrm{H}\left(\frac{a \alpha+b}{c}\right) \leq 2^{n+1} \mathrm{H}(\alpha) \max \{|a|,|b|,|c|\}^{n} .
$$

Proof. Denoting by $P(X)$ the minimal defining polynomial of $\alpha$, we see that $Q(X):=a^{n} P(c X / a-b / a)$ is the one of $(a \alpha+b) / c$. Since the height of $Q(X)$ is bounded from above by $2^{n+1} \mathrm{H}(\alpha) \max \{|a|,|b|,|c|\}^{n}$, the lemma is proved.

LEMma 5 ([1, Lemma 4]). Let $n$ be a positive integer and let $g$ be a prime number with $g>n$. Let $P(X)$ be a monic polynomial of degree $n$ with integer coefficients. Then there is no integer a such that $g$ divides each of $P(a), P(a+1), \ldots, P(a+n)$.

Lemma 6. Let $P(X):=a_{n}\left(X-\alpha_{1}\right) \ldots\left(X-\alpha_{n}\right)$ be a polynomial with complex coefficients of degree $n$ and whose leading coefficient $a_{n}$ is a positive number. Let $\psi>0$ and $\xi$ be real numbers. Then there exist effective positive constants $c_{1}(n, \xi, \psi)$ and $c_{2}(n, \xi, \psi)$, depending only on $n, \psi$ and $\xi$, such that

$$
c_{1}(n, \xi, \psi) \frac{\mathrm{H}(P)}{a_{n}} \leq \prod_{\left|\xi-\alpha_{j}\right| \geq \psi}\left|\xi-\alpha_{j}\right| \leq c_{2}(n, \xi, \psi) \frac{\mathrm{H}(P)}{a_{n}} .
$$

Proof. This follows from Hilfssatz 2 of Wirsing [16].

5. The inductive construction. Theorem 3 below gives an explicit inductive construction of sequences $\left(\xi_{j}\right)_{j \geq 1}$ of real algebraic numbers of degree $n$. It will be proved in Section 6 that such sequences converge to real numbers having the property stated in Theorem 1 . We use in Theorem 3 the same notation as in Lemma 3 , namely we denote by $\delta^{+}(n, a)$ the root of the polynomial $P_{n, a}(X)$ defined in that lemma.

THEOREM 3. Let $n \geq 3$ be an integer and let $\mu, \nu$ be real numbers with $0 \leq \mu \leq(n-2) / 2$ and $\nu>1$. Set $G(n)=2 n^{3}+1$ and let $\chi>G(n)$ be a real number. Then there exist a positive number $\lambda<1 / 2$, prime numbers $g_{1} \geq 11, g_{2}, \ldots$ and integers $c_{1}, c_{2}, \ldots$ such that the following conditions are satisfied, where we have set $\gamma_{j}:=\delta^{+}\left(n,\left[g_{j}^{\mu}\right]\right)$ for any integer $j \geq 1$ :

$g_{j}$ does not divide the norm of $c_{j}+\gamma_{j}(j \geq 1)$.

$\left(\mathrm{II}_{j}\right) \quad \xi_{j}=\left(c_{j}+\gamma_{j}\right) / g_{j}$ belongs to the interval $I_{j-1}$ defined by

$$
\xi_{j-1}+\frac{1}{2} g_{j-1}^{-\nu}<x<\xi_{j-1}+\frac{3}{4} g_{j-1}^{-\nu} \quad(j \geq 2) \text {. }
$$

$\left(\mathrm{III}_{1}\right) \quad\left|\xi_{1}-\alpha\right| \geq 2 \lambda \mathrm{H}(\alpha)^{-\chi}$ for any algebraic number $\alpha \neq \xi_{1}$ of degree $\leq n$. 
$\left(\mathrm{III}_{j}\right) \quad\left|\xi_{j}-\alpha\right| \geq \lambda \mathrm{H}(\alpha)^{-\chi}$ for any algebraic number $\alpha \notin\left\{\xi_{1}, \ldots, \xi_{j}\right\}$ of degree $\leq n(j \geq 2)$.

As will be seen in Section 6, Theorem 3 covers the range of values $[(n-1) / n, n / 4]$ for the function $w_{n}-w_{n}^{*}$. For the interval $[0,(n-1) / n]$, we need Theorem 3 ' below.

Theorem $3^{\prime}$. Theorem 3 holds with the function $G(n)$ replaced by $H(n)$ $=2 n^{3}+2 n^{2}+2 n+1$ and $\gamma_{j}$ by $2^{1 / n}\left[g_{j}^{\mu^{\prime}}\right]$, where $\mu^{\prime}$ is any number in $[0,1]$.

We observe that the sequence $\left(\xi_{j}\right)_{j \geq 1}$ obtained in Theorem 3 is strictly increasing and bounded, hence it converges to a limit $\xi$. For any $j \geq 1$, we have $c_{j} \leq 2 g_{j}$, thus, by Lemma 4 and the definition of $\gamma_{j}$, the height of $\xi_{j}$ satisfies

$$
\mathrm{H}\left(\xi_{j}\right) \leq c(n) g_{j}^{2 n-2},
$$

for some constant $c(n)$ depending only on $n$. Condition $\left(\mathrm{II}_{j+1}\right)$ then shows that the order of approximation of $\xi$ by the algebraic number $\xi_{j}$ depends only on $\nu$ and $n$. Further, conditions ( $\left.\mathrm{III}_{j}\right)$ imply that the other algebraic numbers of degree at most $n$ are not too close to $\xi$. Hence, the precise order of approximation of $\xi$ by algebraic numbers of degree at most $n$ is controlled in terms of $n, \nu$ and $\chi$.

The rôle of the parameter $\mu$ is to measure the gap between $w_{n}(\xi)$ and $w_{n}^{*}(\xi)$, as will be shown in Section 5 .

To simplify the notation, in what follows we denote by $\alpha$ a real algebraic number of degree less than or equal to $n$. Let $\varepsilon$ be a positive number such that

$$
\chi>2 n^{3}+1+2 n^{2} \varepsilon .
$$

In order to prove Theorem 3 , we add three extra conditions $\left(\mathrm{IV}_{j}\right),\left(\mathrm{V}_{j}\right)$ and $\left(\mathrm{VI}_{j}\right)$, which should be satisfied by the numbers $\xi_{j}$. We denote by Leb the Lebesgue measure on the real line.

Let $J_{j}$ denote the subset of $I_{j}$ consisting of the real numbers $x \in I_{j}$ satisfying

$$
|x-\alpha| \geq 2 \lambda \mathrm{H}(\alpha)^{-\chi}
$$

for any algebraic number $\alpha$ of degree $\leq n$, distinct from $\xi_{1}, \ldots, \xi_{j}, x$ and of height $\mathrm{H}(\alpha)$ sufficiently large, that is, satisfying

$$
\mathrm{H}(\alpha) \geq\left(\lambda g_{j}^{\nu}\right)^{1 / \chi} .
$$

The supplementary conditions are the following:

$\left(\mathrm{IV}_{j}\right) \quad \xi_{j} \in J_{j-1}(j \geq 2)$.

$\left(\mathrm{V}_{j}\right) \quad$ If $\mathrm{H}(\alpha) \leq g_{j}^{1 /(n+1+\varepsilon)}$, then $\left|\xi_{j}-\alpha\right| \geq 1 / g_{j}(j \geq 1)$.

$\left(\mathrm{VI}_{j}\right) \quad$ The measure of $J_{j}$ satisfies $\operatorname{Leb}\left(J_{j}\right) \geq \operatorname{Leb}\left(I_{j}\right) / 2(j \geq 1)$. 
We construct the numbers $\xi_{1}, \xi_{2}, \ldots$ by induction. At the $j$ th stage, there are two distinct steps. Step $\left(\mathrm{A}_{j}\right)$ consists in building an algebraic number $\xi_{j}=\left(c_{j}+\gamma_{j}\right) / g_{j}$ of degree $n$ satisfying conditions $\left(\mathrm{I}_{j}\right)$ to $\left(\mathrm{V}_{j}\right)$. In step $\left(\mathrm{B}_{j}\right)$, we show that the number $\xi_{j}$ constructed in $\left(\mathrm{A}_{j}\right)$ satisfies $\left(\mathrm{VI}_{j}\right)$ as well, provided that $g_{j}$ is chosen large enough in terms of

$$
n, \mu, \nu, \chi, \varepsilon, \lambda, \xi_{1}, \ldots, \xi_{j-1} \text {. }
$$

The symbols $o$, $\gg$ and $\ll$ used throughout steps $\left(\mathrm{A}_{j}\right)$ and $\left(\mathrm{B}_{j}\right)$ mean that the numerical implicit constants depend (at most) on the quantities (4). Furthermore, the symbol $o$ implies "as $g_{j}$ tends to infinity".

Step $\left(\mathrm{A}_{1}\right)$ is rather easy. Let $P(X)$ denote the minimal defining polynomial of $\gamma_{1}$ and observe that $\left(\mathrm{I}_{1}\right)$ is satisfied if, and only if, $g_{1}$ does not divide $P\left(-c_{1}\right)$. Thus, by Lemma 5 , if the prime number $g_{1}$ is larger than $n$, then there are $\gg g_{1}$ numbers $\xi_{1}=\left(c_{1}+\gamma_{1}\right) / g_{1}$ in the interval ]1,2[ satisfying condition $\left(\mathrm{I}_{1}\right)$. These $\gg g_{1}$ numbers have mutual distances at least $g_{1}^{-1}$, and since there are only $o\left(g_{1}\right)$ algebraic numbers $\alpha$ of degree at most $n$ satisfying $\mathrm{H}(\alpha) \leq g_{1}^{1 /(n+1+\varepsilon)}$, one can choose $\xi_{1}$ such that $\left(\mathrm{V}_{1}\right)$ is satisfied. We point out that there are $\gg g_{1}$ choices for $c_{1}$, where the constant implied in $\gg$ depends only on $n$. Further, by Lemma 2, we have

$$
\left|\xi_{1}-\alpha\right| \geq 2 \lambda \mathrm{H}(\alpha)^{-n}
$$

with $\lambda=c(n, n) \mathrm{H}\left(\xi_{1}\right)^{-n} / 2$, for any real algebraic numbers $\alpha \neq \xi_{1}$ of degree at most $n$. Thus $\left(\mathrm{I}_{1}\right),\left(\mathrm{II}_{1}\right),\left(\mathrm{III}_{1}\right)$ and $\left(\mathrm{V}_{1}\right)$ are satisfied.

Let $j \geq 2$ be an integer and assume that $\xi_{1}, \ldots, \xi_{j-1}$ have been constructed. Step $\left(\mathrm{A}_{j}\right)$ is much harder to verify, since we have no control on the set $J_{j-1}$. Thus, it seems difficult to check that condition $\left(\mathrm{IV}_{j}\right)$ holds. To overcome this problem, we follow Schmidt's argument [11], also used by Baker [1]. We set $\xi_{j}=\left(c_{j}+\gamma_{j}\right) / g_{j}$ for some positive integers $c_{j}$ and $g_{j}>8 g_{j-1}$ and we introduce the set $J_{j-1}^{\prime}$ formed by the real numbers $x \in I_{j-1}$ satisfying

$$
|x-\alpha| \geq 2 \lambda \mathrm{H}(\alpha)^{-\chi}
$$

for any algebraic number $\alpha$ of degree $\leq n$, distinct from $\xi_{1}, \ldots, \xi_{j}, x$, and whose height $\mathrm{H}(\alpha)$ satisfies the inequalities

$$
\left(\lambda g_{j-1}^{\nu}\right)^{1 / \chi} \leq \mathrm{H}(\alpha) \leq\left(c_{2}(n)^{-1} g_{j}^{2 n(n-1)}\right)^{1 /(\chi-n)} .
$$

Since, by (3), we have

$$
\chi-n>2 n(n-1)(n+1),
$$

the exponent of $g_{j}$ in the right member of (5) is strictly less than $1 /(n+1)$. Thus, there are $o\left(g_{j}\right)$ algebraic numbers $\alpha$ satisfying (5), and we observe that, unlike $J_{j-1}$, the set $J_{j-1}^{\prime}$ is a finite union of intervals, and more precisely, a union of $o\left(g_{j}\right)$ intervals. 
We will prove that for $g_{j}$ large enough we have $\gg g_{j}$ choices for $c_{j}$ in order that conditions $\left(\mathrm{I}_{j}\right)$ to $\left(\mathrm{V}_{j}\right)$ are satisfied.

Let $\alpha$ be an algebraic number of degree $\leq n$. Since $\mathrm{H}\left(\gamma_{j}\right) \leq 2 g_{j}^{n-2}$ and $c_{j} \leq 2 g_{j}$, we infer from Lemmas 2 and 4 that there exist positive constants $c_{1}(n)$ and $c_{2}(n)$ such that

$$
\left|\xi_{j}-\alpha\right| \geq c_{1}(n) \mathrm{H}\left(\xi_{j}\right)^{-n} \mathrm{H}(\alpha)^{-n} \geq c_{2}(n) g_{j}^{-2 n(n-1)} \mathrm{H}(\alpha)^{-n} .
$$

In particular, using $2 \lambda<1$, we have

$$
\left|\xi_{j}-\alpha\right| \geq 2 \lambda \mathrm{H}(\alpha)^{-\chi}
$$

as soon as

$$
\mathrm{H}(\alpha)^{\chi-n} \geq c_{2}(n)^{-1} g_{j}^{2 n(n-1)} .
$$

By $\left(\mathrm{VI}_{j-1}\right)$ and since $J_{j-1}^{\prime} \supset J_{j-1}$, we have $\operatorname{Leb}\left(J_{j-1}^{\prime}\right) \gg 1$. Since the set $J_{j-1}^{\prime}$ is the union of $o\left(g_{j}\right)$ intervals, if $g_{j}$ is a sufficiently large prime number, then, using Lemma 5 as in step $\left(\mathrm{A}_{1}\right)$, we find that there exist $\gg g_{j}$ numbers $\xi_{j}=\left(c_{j}+\gamma_{j}\right) / g_{j}$ in $J_{j-1}^{\prime}$ such that $\left(\mathrm{I}_{j}\right)$ is satisfied. Such $\xi_{j}$ 's also belong to $J_{j-1}$, since (8) implies (7), and condition $\left(\mathrm{IV}_{j}\right)$ is satisfied.

Thus, we are left with $\gg g_{j}$ suitable algebraic numbers $\xi_{j}$, mutually distant by at least $g_{j}^{-1}$. Only $o\left(g_{j}\right)$ algebraic numbers $\alpha$ of degree at most $n$ satisfy

$$
\mathrm{H}(\alpha) \leq g_{j}^{1 /(n+1+\varepsilon)},
$$

thus one can choose $\xi_{j}$ in such a way that $\left|\xi_{j}-\alpha\right| \geq 1 / g_{j}$ for the numbers $\alpha$ satisfying (9). Consequently, there are $\gg g_{j}$ algebraic numbers $\xi_{j}$ satisfying $\left(\mathrm{I}_{j}\right),\left(\mathrm{II}_{j}\right),\left(\mathrm{IV}_{j}\right)$ and $\left(\mathrm{V}_{j}\right)$.

It remains to show that such a $\xi_{j}$ also satisfies $\left(\mathrm{III}_{j}\right)$. To this end, it suffices to prove that

$$
\left|\xi_{j}-\alpha\right| \geq \lambda \mathrm{H}(\alpha)^{-\chi}
$$

for the algebraic numbers $\alpha$ of degree $\leq n$ which are different from $\xi_{1}, \ldots, \xi_{j}$ and whose height $\mathrm{H}(\alpha)$ satisfies

$$
\mathrm{H}(\alpha)<\left(\lambda g_{j-1}^{\nu}\right)^{1 / \chi}
$$

Since the sequence $\left(g_{t}\right)_{t \geq 1}$ is increasing, either we have

$$
g_{1}^{-\nu}<\lambda \mathrm{H}(\alpha)^{-\chi},
$$

or there exists an integer $t$ with $2 \leq t<j$ such that

$$
g_{t}^{-\nu}<\lambda \mathrm{H}(\alpha)^{-\chi} \leq g_{t-1}^{-\nu} \text {. }
$$

In the former case, we infer from $\left(\mathrm{III}_{1}\right)$ and $(10)$ that

$$
\left|\xi_{j}-\alpha\right| \geq\left|\xi_{1}-\alpha\right|-\left|\xi_{j}-\xi_{1}\right| \geq 2 \lambda \mathrm{H}(\alpha)^{-\chi}-g_{1}^{-\nu}>\lambda \mathrm{H}(\alpha)^{-\chi} .
$$


In the latter case, $\left(\mathrm{IV}_{t}\right)$ and (11) yield

$$
\left|\xi_{j}-\alpha\right| \geq\left|\xi_{t}-\alpha\right|-\left|\xi_{j}-\xi_{t}\right| \geq 2 \lambda \mathrm{H}(\alpha)^{-\chi}-g_{t}^{-\nu}>\lambda \mathrm{H}(\alpha)^{-\chi} .
$$

The upper estimates $\left|\xi_{j}-\xi_{1}\right| \leq g_{1}^{-\nu}$ and $\left|\xi_{j}-\xi_{t}\right| \leq g_{t}^{-\nu}$ used above follow from $\left(\mathrm{II}_{j}\right)$ and the assumption $g_{l}>8 g_{l-1}$, valid for any integer $l$ with $2 \leq$ $l \leq j$. Consequently, condition $\left(\mathrm{III}_{j}\right)$ holds and the proof of step $\left(\mathrm{A}_{j}\right)$ is complete.

Let $j \geq 1$ be an integer. For the proof of step $\left(\mathrm{B}_{j}\right)$, we first establish that if $g_{j}$ is large enough and if $x$ lies in $I_{j}$, then

$$
|x-\alpha| \geq 2 \lambda \mathrm{H}(\alpha)^{-\chi}
$$

for any algebraic number $\alpha \neq \xi_{j}$ such that

$$
\left(\lambda g_{j}^{\nu}\right)^{1 / \chi} \leq \mathrm{H}(\alpha) \leq g_{j}^{\nu /(\chi-n-1-\varepsilon)} .
$$

Let then $\alpha \neq \xi_{j}$ be an algebraic number of degree $\leq n$ satisfying (13) and let $x$ be in $I_{j}$, that is,

$$
\frac{1}{2} g_{j}^{-\nu}<x-\xi_{j}<\frac{3}{4} g_{j}^{-\nu} .
$$

If $g_{j}^{\nu /(\chi-n-1-\varepsilon)} \leq g_{j}^{1 /(n+1+\varepsilon)}$, then $\mathrm{H}(\alpha) \leq g_{j}^{1 /(n+1+\varepsilon)}$ and it follows from $\left(\mathrm{V}_{j}\right),(13)$ and $(14)$ that

$$
|x-\alpha| \geq\left|\xi_{j}-\alpha\right|-\left|\xi_{j}-x\right| \geq g_{j}^{-1}-g_{j}^{-\nu} \geq 2 g_{j}^{-\nu} \geq 2 \lambda \mathrm{H}(\alpha)^{-\chi} .
$$

Otherwise, we have

$$
g_{j}^{\nu /(\chi-n-1-\varepsilon)}>g_{j}^{1 /(n+1+\varepsilon)},
$$

and, by (6), we get

$$
\begin{aligned}
|x-\alpha| & \geq\left|\xi_{j}-\alpha\right|-\left|\xi_{j}-x\right| \geq c_{2}(n) g_{j}^{-2 n(n-1)} \mathrm{H}(\alpha)^{-n}-g_{j}^{-\nu} \\
& \geq c_{2}(n) g_{j}^{-2 n(n-1)} \mathrm{H}(\alpha)^{-n} / 2 .
\end{aligned}
$$

To check the last inequality, we have to verify that

$$
2 g_{j}^{-\nu} \leq c_{2}(n) g_{j}^{-2 n(n-1)} \mathrm{H}(\alpha)^{-n} .
$$

In view of $(13),(18)$ is true as soon as

$$
2 g_{j}^{n \nu /(\chi-n-1-\varepsilon)} \leq c_{2}(n) g_{j}^{\nu} g_{j}^{-2 n(n-1)},
$$

which, by (14), holds for $g_{j}$ large enough when

$$
\frac{n}{\chi-n-1-\varepsilon}<1-2 n(n-1) \frac{n+1+\varepsilon}{\chi-n-1-\varepsilon},
$$

in particular when $\chi$ satisfies (3).

Moreover, we have

$$
c_{2}(n) g_{j}^{-2 n(n-1)} \mathrm{H}(\alpha)^{-n} \geq 4 \lambda \mathrm{H}(\alpha)^{-\chi} .
$$


Indeed, by (13), $\lambda<1$ and (16), we get

$$
\begin{aligned}
\mathrm{H}(\alpha)^{\chi-n} & \geq\left(\lambda g_{j}^{\nu}\right)^{(\chi-n) / \chi} \geq \lambda g_{j}^{(\chi-n)(\chi-n-1-\varepsilon) /(\chi n+\chi+\chi \varepsilon)} \\
& \geq 4 \lambda c_{2}(n)^{-1} g_{j}^{2 n(n-1)},
\end{aligned}
$$

since we infer from (3) that

$$
(\chi-n)(\chi-n-1-\varepsilon)>2 \chi n(n+1+\varepsilon)(n-1) .
$$

Combining (17) and (19), we have checked that

$$
|x-\alpha| \geq 2 \lambda \mathrm{H}(\alpha)^{-\chi}
$$

when (16) holds; hence, by (15), (12) is true if $\alpha \neq \xi_{j}$ satisfies (13). Consequently, if $g_{j}$ is large enough, then the complement $J_{j}^{\mathrm{c}}$ of $J_{j}$ in $I_{j}$ is contained in the union of the intervals

$$
E(\alpha):=] \alpha-2 \lambda \mathrm{H}(\alpha)^{-\chi}, \alpha+2 \lambda \mathrm{H}(\alpha)^{-\chi}[,
$$

where $\alpha$ runs over the set of algebraic numbers of degree $\leq n$ and with height greater than $g_{j}^{\nu /(\chi-n-1-\varepsilon)}$. The Lebesgue measure of $J_{j}^{\text {c }}$ is then

$$
\ll \sum_{H>g_{j}^{\nu /(\chi-n-1-\varepsilon)}} H^{n-\chi}=o\left(g_{j}^{-\nu}\right)=o\left(\operatorname{Leb}\left(I_{j}\right)\right) .
$$

Thus, we conclude that we can find $g_{j}$ large enough such that $\operatorname{Leb}\left(J_{j}\right) \geq$ $\operatorname{Leb}\left(I_{j}\right) / 2$. This completes step $\left(\mathrm{B}_{j}\right)$ as well as the proof of Theorem 3 .

The proof of Theorem $3^{\prime}$ follows the same lines as that of Theorem 3 , the only difference being that the estimate $\mathrm{H}\left(\gamma_{j}\right) \leq 2 g_{j}^{n}$ should replace $\mathrm{H}\left(\gamma_{j}\right) \leq 2 g_{j}^{n-2}$. Thus, the assumption (3) should be modified and we have to replace $G(n)$ by $H(n)$.

6. Completion of the proof of Theorem 1. We first deal with the range of values $[(n-1) / n, n / 4]$. Let $\Delta$ be in $[(n-1) / n, n / 4]$ and set

$$
\mu=\frac{2(n \Delta-n+1)}{n-2} \text {. }
$$

We observe that $\mu$ is in $[0,(n-2) / 2]$. Let $w_{n}>2 n^{3}+n-1$ and set $w_{n}^{*}=$ $w_{n}-\Delta$. The sequence $\left(\xi_{j}\right)_{j \geq 1}$ obtained in Theorem 3 is strictly increasing and bounded, thus it converges towards a real number denoted by $\xi$. Set $\nu=n\left(w_{n}^{*}+1\right)$ and set $\chi=w_{n}-n+2$ so that $\chi>2 n^{3}+1$. Let $\xi_{1}, \xi_{2}, \ldots$ be as in Theorem 3 and denote by $\xi$ the limit of the strictly increasing sequence $\left(\xi_{j}\right)_{j \geq 1}$.

We write $A \ll B$ if there exists a constant $c(n)$, depending only on $n$, such that $|A|<c(n) B$, and we write $A \asymp B$ if both $A \ll B$ and $B \ll A$.

Our choice of $\gamma_{j}$ implies that the minimal defining polynomial of $\xi_{j}$ is

$$
Q_{j}(X):=\left(g_{j} X-c_{j}\right)^{n}-2\left(\left[g_{j}^{\mu}\right]\left(g_{j} X-c_{j}\right)-1\right)^{2} .
$$


This polynomial is indeed irreducible and primitive by $\left(\mathrm{I}_{j}\right)$ and the first statement of Lemma 3 . Since $\mu \leq(n-2) / 2$, we have $\mathrm{H}\left(\xi_{j}\right) \asymp g_{j}^{n}$.

Moreover, for any $j \geq 1$,

$$
\xi_{j}+g_{j}^{-\nu} / 2<\xi<\xi_{j}+g_{j}^{-\nu}
$$

and we deduce that

$$
\left|\xi-\xi_{j}\right| \asymp \mathrm{H}\left(\xi_{j}\right)^{-\nu / n} \asymp \mathrm{H}\left(\xi_{j}\right)^{-w_{n}^{*}-1} .
$$

Further, if $\alpha$ is of degree $\leq n$ and is not one of the $\xi_{j}$ 's, then $|\xi-\alpha| \geq$ $\lambda \mathrm{H}(\alpha)^{-\chi}$, whence

$$
|\xi-\alpha| \geq \mathrm{H}(\alpha)^{-w_{n}^{*}-1},
$$

since $\chi \leq w_{n}^{*}+1$. It follows from $(20),(21)$ and Lemma 1 that $w_{n}^{*}(\xi)=w_{n}^{*}$.

It now remains to prove that $w_{n}(\xi)=w_{n}$. Denote by $\xi_{j}=\beta_{j 1}, \ldots, \beta_{j n}$ the roots of $Q_{j}(X)$, numbered in such a way that $\left|\xi_{j}-\beta_{j 2}\right| \asymp g_{j}^{-\mu(n+2) / 2-1}$. Denote by $\delta_{3}, \ldots, \delta_{k}$ the roots of $P_{n,\left[g_{j}^{\mu}\right]}(X)$ other than $\delta^{+}\left(n,\left[g_{j}^{\mu}\right]\right)$ and $\delta^{-}\left(n,\left[g_{j}^{\mu}\right]\right)$. For $k \geq 3$, we have

$$
\left|\xi_{j}-\beta_{j k}\right| \asymp \frac{\left|1 /\left[g_{j}^{\mu}\right]-\delta_{k}\right|}{g_{j}} .
$$

Consequently, we get

$$
\begin{aligned}
\left|Q_{j}(\xi)\right| & =g_{j}^{n}\left|\xi-\xi_{j}\right|\left|\xi-\beta_{j 2}\right| \prod_{3 \leq k \leq n}\left|\xi-\beta_{j k}\right| \\
& \asymp g_{j}^{2} \mathrm{H}\left(\xi_{j}\right)^{-w_{n}^{*}-1} g_{j}^{-\mu(n+2) / 2-1} \prod_{3 \leq k \leq n}\left|1 / a-\delta_{k}\right| \\
& \asymp \mathrm{H}\left(\xi_{j}\right)^{-w_{n}^{*}-1} g_{j}^{-\mu(n+2) / 2+1}\left[g_{j}^{\mu}\right]^{2},
\end{aligned}
$$

by Lemma 6 and the last statement of Lemma 3 . Thus,

$$
\left|Q_{j}(\xi)\right| \asymp \mathrm{H}\left(Q_{j}\right)^{-w_{n}^{*}-1-\mu(n-2) /(2 n)+1 / n},
$$

and we see that

$$
w_{n}(\xi) \geq w_{n}^{*}+1+\mu(n-2) /(2 n)-1 / n
$$

hence, by definition of $\mu$, we obtain

$$
w_{n}(\xi) \geq w_{n}^{*}+\Delta
$$

In order to show that the inequalities in (22) and (23) are indeed equalities, we argue exactly as in Baker [1]. Let $P(X)$ be an integer polynomial of degree $\leq n$ which is not a multiple of some $Q_{j}(X)$. Write

$$
P(X)=a R_{1}(X) \ldots R_{p}(X),
$$

where $a$ is an integer and the polynomials $R_{i}(X)$ are primitive and irreducible. Since $R_{i}(\xi) \neq 0$, if $k$ denotes the degree of the polynomial $R_{i}(X)$, 
then, by $[4$, Theorem 4], this polynomial has a root $\theta$ satisfying

$$
\left|R_{i}(\xi)\right| \gg \mathrm{H}\left(R_{i}\right)^{2-k}|\xi-\theta| \gg \lambda \mathrm{H}\left(R_{i}\right)^{-\chi-k+2} \gg \lambda \mathrm{H}\left(R_{i}\right)^{-w_{n}} .
$$

Consequently, it follows from (24) and the "Gelfond inequality" (see e.g. [15, Remark 2, p. 81]) that

$$
|P(\xi)| \gg\left(\mathrm{H}\left(R_{1}\right) \ldots \mathrm{H}\left(R_{p}\right)\right)^{-w_{n}} \gg \mathrm{H}(P)^{-w_{n}},
$$

and we get $w_{n}(\xi)=w_{n}$, as claimed.

We now consider $\Delta^{\prime}$ in the range $[0,(n-1) / n]$ and set

$$
\mu^{\prime}=\frac{n-1-n \Delta^{\prime}}{n-1} .
$$

We argue as above except that we use Theorem $3^{\prime}$ instead of Theorem 3 . The polynomials $Q_{j}(X)$ are replaced by

$$
R_{j}(X):=\left(g_{j} X-c_{j}\right)^{n}-2\left[g_{j}^{\mu^{\prime}}\right]^{n},
$$

which have been used by Baker [1], and, proceeding as above, we show that

$$
\left|R_{j}(\xi)\right| \asymp g_{j}^{n}\left|\xi-\xi_{j}\right| g_{j}^{(\mu-1)(n-1)} \asymp \mathrm{H}\left(R_{j}\right)^{-w_{n}^{*}-(1-\mu)(n-1) / n}
$$

and

$$
w_{n}(\xi)=w_{n}^{*}(\xi)+(1-\mu) \frac{n-1}{n}=w_{n}^{*}(\xi)+\Delta^{\prime} .
$$

The proof of Theorem 1 is now finished.

7. Outline of the proof of Theorem 2. As pointed out at the end of steps $\left(\mathrm{A}_{1}\right)$ and $\left(\mathrm{A}_{j}\right)$, the integers $c_{j}$ occurring in the inductive construction of Theorem 3 are far from being uniquely determined. Indeed, as stated by Baker [1, p. 29], it turns out that, if $g_{j}$ is sufficiently large, we have at each step $\left(\mathrm{A}_{j}\right)$ with $j \geq 2$ at least

$$
\frac{g_{j} g_{j-1}^{-\nu}}{32 n}
$$

suitable choices for $\xi_{j}$. Observe that $g_{j-1}^{-\nu} / 4$ is the length of the interval $I_{j-1}$ and that the $n$ occurring in the denominator of (25) is a consequence of Lemma 5. No particular importance has to be attached to the constant 32. This shows that we obtain an uncountable set of real numbers $\xi$ with the property stated in Theorem 1. Moreover, using the method described in Section 5 of [1], it can be shown that they form a set with positive $h$-measure for some function $h: t \mapsto t^{\delta}$ with $\delta>0$ (in [1], the $\nu_{j}^{\prime}$ 's are unbounded, thus $h$ has to grow faster than any function $t \mapsto t^{\delta}$ in a neighborhood of the origin), thus with positive Hausdorff dimension. Since the sets of $T$ - and $U$-numbers have Hausdorff dimension zero (see e.g. [5]), it follows that, for any fixed integer $n \geq 2$, there exist $S$-numbers $\xi$ with the property stated in Theorem 1. 
In order to control simultaneously finitely many $w_{n}(\xi)$ and $w_{n}^{*}(\xi)$, only slight modifications of Theorem 3 are needed. Essentially, it is sufficient to use at the $j$ th inductive step the algebraic number $\gamma_{j}:=\delta^{+}\left(n_{l+1},\left[g_{j}^{\mu}\right]\right)$, where $l$ is the remainder in the Euclidean division of $j$ by $k$.

\section{Further remarks}

- Comparison with the work of Baker. Let $n \geq 1$ be an integer and let $w_{n}^{*}$ be a given real number. Clearly, it is an easy matter to construct a real number $\xi$ with $w_{n}^{*}(\xi) \geq w_{n}^{*}$, e.g. by using a nested interval construction. However, it is much more difficult to get an upper bound for $w_{n}^{*}(\xi)$, and in particular to ensure that $w_{n}^{*}(\xi)=w_{n}^{*}$.

Theorem 1 of Baker [1] depends on the following deep result of Schmidt $[11,12]$.

Theorem S. Let $\beta$ be a real algebraic number and let $\eta>0$ be real. Let $n \geq 1$ be an integer. Then there exists an ineffective positive constant $C_{1}(\beta, n, \eta)$, depending only on $\beta, n$ and $\eta$, such that

$$
|\beta-\alpha| \geq C_{1}(\beta, n, \eta) \mathrm{H}(\alpha)^{-n-1-\eta}
$$

for all real algebraic numbers $\alpha \neq \beta$ of degree at most $n$.

Wirsing [17] obtained a slightly weaker result (with $-2 n-\eta$ in the exponent of $\mathrm{H}(\alpha)$ ), which turns out to be sufficient to confirm the existence of $T$-numbers (see $[10,11]$ ). Indeed, the crucial point is that the exponent of $\mathrm{H}(\alpha)$ in $(26)$ does not depend on $\beta$.

If we use Theorem $\mathrm{S}$ with $\eta=1$ instead of Lemma 2 in (6), we deduce that there exists a constant $c\left(\gamma_{j}\right)$ such that

$$
\left|\xi_{j}-\alpha\right|=\frac{1}{g_{j}}\left|\gamma_{j}-\left(g_{j} \alpha-c_{j}\right)\right| \geq c\left(\gamma_{j}\right) g_{j}^{-n^{2}-1} \mathrm{H}(\alpha)^{-n-2} .
$$

However, $\gamma_{j}$ depends on $g_{j}$, and we do not have any estimate for $c\left(\gamma_{j}\right)$. Thus we cannot argue as in (7), (8), etc. Consequently, the method used in the present paper does not allow us to construct real numbers $\xi$ satisfying the conclusion of Theorem 1 for every integer $n \geq 3$.

In his paper, Baker used for $\gamma_{j}$ the numbers $2^{1 / n}\left[g_{j}^{\mu}\right]$, where $\mu$ runs over $[0,1]$ and allows him to control the difference between $w_{n}$ and $w_{n}^{*}$. In this case, Theorem S can be applied since

$$
\begin{aligned}
\left|\xi_{j}-\alpha\right| & =\frac{1}{g_{j}}\left|2^{1 / n}\left[g_{j}^{\mu}\right]-\left(g_{j} \alpha-c_{j}\right)\right|=\frac{\left[g_{j}^{\mu}\right]}{g_{j}}\left|2^{1 / n}-\frac{g_{j} \alpha-c_{j}}{\left[g_{j}^{\mu}\right]}\right| \\
& \geq c\left(2^{1 / n}\right) g_{j}^{-n^{2}-2 n-1} \mathrm{H}(\alpha)^{-n-2} .
\end{aligned}
$$


Here, $c\left(2^{1 / n}\right)$ does not depend on $j$, thus one can argue as in $(7),(8)$, etc. The exponent of $g_{j}$ in (27) is slightly larger than in (6), thus we get a better lower bound for $\chi$, whose order of magnitude is $n^{3}$, however.

- Approximation in the complex field. It is possible to adapt the proof of Theorem 1 to construct complex non-real numbers $\xi$ with $w_{n}^{*}(\xi)<w_{n}(\xi)$, as Baker [1] did in his Theorem 2. Our method allows us to show that for any integer $n \geq 3$ the set of values taken by the function $w_{2 n}-w_{2 n}^{*}$ evaluated at complex non-real numbers contains the interval $[0, n / 8]$. Presumably, it should be possible to show that non-real numbers $\xi$ with $w_{n}^{*}(\xi)<w_{n}(\xi)$ for some odd integer $n \geq 5$ exist; the problem is to find suitable polynomials to replace $P_{n, a}(X)$.

- Approximation in p-adic fields. Presumably, the method can be carried over to the $p$-adic field $\mathbb{Q}_{p}$ without too much difficulty in order to prove that there exist $p$-adic numbers $\xi$ with $w_{n}(\xi) \neq w_{n}^{*}(\xi)$. The polynomials $X^{n}-2\left(X-p^{a}\right)^{2}$ would then play the rôle of the polynomials $P_{n, a}(X)$ defined in Lemma 3.

\section{References}

[1] R. C. Baker, On approximation with algebraic numbers of bounded degree, Mathematika 23 (1976), 18-31.

[2] P. Borwein and T. Erdélyi, Polynomials and Polynomial Inequalities, Grad. Texts in Math. 161, Springer, 1995.

[3] K. Falconer, Fractal Geometry: Mathematical Foundations and Applications, Wiley, 1990.

[4] R. Güting, Polynomials with multiple zeros, Mathematika 14 (1967), 149-159.

[5] F. Kasch und B. Volkmann, Zur Mahlerschen Vermutung über S-Zahlen, Math. Ann. 136 (1958), 442-453.

[6] J. F. Koksma, Über die Mahlersche Klasseneinteilung der transzendenten Zahlen und die Approximation komplexer Zahlen durch algebraische Zahlen, Monatsh. Math. Phys. 48 (1939), 176-189.

[7] K. Mahler, Zur Approximation der Exponentialfunktionen und des Logarithmus. I, II, J. Reine Angew. Math. 166 (1932), 118-150.

[8] M. Mignotte, Some useful bounds, in: Computer Algebra, B. Buchberger, G. E. Collins and R. Loos (eds.), Springer, 1982, 259-263.

[9] A. M. Robert, A Course in p-adic Analysis, Grad. Texts in Math. 198, Springer, New York, 2000.

[10] W. M. Schmidt, T-numbers do exist, in: Symposia Math. IV, Inst. Naz. di Alta Math. (Rome, 1968), Academic Press, 1970, 3-26.

[11] -, Mahler's T-numbers, in: Proc. Sympos. Pure Math. 20, Amer. Math. Soc., Providence, RI, 1971, 275-286.

[12] - Approximation to Algebraic Numbers, Monograph. Enseign. Math. 19, Genève, 1971.

[13] -, Diophantine Approximations, Lecture Notes in Math. 785, Springer, Berlin, 1980. 
[14] T. Schneider, Introduction aux nombres transcendants, Gauthier-Villars, Paris, 1959.

[15] M. Waldschmidt, Diophantine Approximation on Linear Algebraic Groups. Transcendence Properties of the Exponential Function in Several Variables, Grundlehren Math. Wiss. 326, Springer, Berlin, 2000.

[16] E. Wirsing, Approximation mit algebraischen Zahlen beschränkten Grades, J. Reine Angew. Math. 206 (1961), 67-77.

[17] - On approximations of algebraic numbers by algebraic numbers of bounded degree, in: Proc. Sympos. Pure Math. 20, Amer. Math. Soc., Providence, RI, 1971, 213-247.

U. F. R. de mathématiques

Université Louis Pasteur

7, rue René Descartes

67084 Strasbourg, France

E-mail: bugeaud@math.u-strasbg.fr

Received on 9.9.2002

and in revised form on 13.1.2003 